DNA Repair (Amst). 2011 November 10; 10(11): 1183-1185. doi:10.1016/j.dnarep.2011.09.003.

\title{
DNA repair inhibitors: Where do we go from here?
}

\author{
Mark R. Kelleya,b,c, \\ aDepartment of Pediatrics (Section of Hematology/Oncology), Herman B Wells Center for \\ Pediatric Research, Indiana University School of Medicine, Indianapolis, IN 46202, United States \\ ${ }^{\text {b}}$ Department of Biochemistry and Molecular Biology, Indiana University School of Medicine, \\ Indianapolis, IN 46202, United States \\ 'Department of Pharmacology and Toxicology, Indiana University School of Medicine, \\ Indianapolis, IN 46202, United States
}

\section{Background}

Although the use of targeted DNA repair inhibitors as cancer therapies is, in many ways, in its infancy, many strides are being made to augment their use and utility. Many challenges exist in doing so, as this involves identifying prognostic and predictive biomarkers, then developing selective DNA repair inhibitors that can act as single agents or potentiators of other anticancer agents. The success of these collective efforts hinges on a deeper understanding of how DNA damage response and repair pathways work in both normal and tumor tissues.

\section{Beyond PARP inhibitors}

The recent extraordinary results obtained in using PARP inhibitors against BRCA-deficient breast cancers was a victory for DNA repair inhibition - a success that has helped propel the research of numerous DNA repair inhibitors into preclinical and clinical development. However, their clinical effectiveness may fall short of expectations unless we can predict which types of cancers will respond best to them. To do that, researchers must identify biomarkers that can reliably pinpoint what types of DNA repair deficiencies various cancers possess. Furthermore, routine tests of gene expression and protein functionality must be developed, to determine prognostic value and predictive treatment success. Having these tools readily available would create a paradigm shift that could revolutionize the structure and outcomes of clinical trials for anticancer treatments.

Treating BRCA-deficient breast cancers through PARP inhibition was an invaluable "find." However, inhibiting a single DNA repair protein or its gene to create a synthetic lethality is probably the exception rather than the rule. Exploiting a cancer-induced DNA repair deficiency by inhibiting just one protein is almost too good to be true. We have much to learn about repair defects associated with cancers, and most of those defects are still undiscovered [1]. We have even more to learn regarding how cancers compensate for those defects using crosstalk and recruitment of alternate pathways through means that yet remain unidentified.

\footnotetext{
(c) 2011 Elsevier B.V. All rights reserved.

*Correspondence address: Department of Pediatrics (Section of Hematology/Oncology), Herman B Wells Center for Pediatric Research, Indiana University School of Medicine, Walther Hall - R3; Rm 528, 980 West Walnut, Indianapolis, IN 46202, United States. Tel.: +1 317274 2755; fax: +1 317274 8046. mkelley@iupui.edu.
} 
Similarly, work to date on identifying cancer biomarkers indicates that most biomarkers will not be single genes, but rather combinations of genes or groups of single nucleotide polymorphisms (SNPs). Identification of epigenetic factors may provide another avenue that can be manipulated for therapeutic benefit; but, again, a combination of those factors will likely be unique to certain cancers. Thus, DNA repair pathways still hold many secrets waiting to be translated into future clinical applications.

\section{Meeting today's challenges}

Three challenges exist in the area of using DNA repair inhibitors to "treat a weakness." We must: (1) identify altered protein expression that is clinically relevant and unique to cancers' dysfunctional DNA repair response, (2) assess how multiple proteins collectively help create and perpetuate a mutagenic phenotype, and (3) determine which inhibitor(s) can confer the greatest therapeutic value with minimal or no collateral damage to normal tissues [2].

Overcoming these challenges could provide the major breakthrough in cancer treatment that has eluded researchers for decades. As Aziz et al. (2010) noted "A thorough understanding of DNA repair pathways dysregulated during carcinogenesis and neoplastic progression, as well as types of DNA damage induced during cancer development, may hold the key to the breakthrough in improving therapy in the fight against cancer" [3].

To most effectively use DNA repair inhibitors and other inhibitors in battling cancer, a paradigm shift in R\&D and patient treatment must take place in a coordinated, comprehensive fashion. This can be described as nine "buckets" of activities.

\subsection{Characterize DNA repair pathways more completely}

More complete knowledge of the molecular interactions of such processes in human cancers and human derived tumor cell lines and not just transformed cell lines is necessary to accurately identify biomarkers and determine which repair proteins to inhibit for the greatest therapeutic benefits. Characterizing these pathways also must include widespread efforts to collect prospective patient samples from populations of healthy people and people with cancer [4].

\subsection{Develop better testing methods for gene expression}

Gene expression profiling for DNA repair activity needs to become more advanced and comprehensive. While investigators can test for a single gene that is known to be altered in a certain cancer, that gene by itself may not accurately represent DNA repair activity. Testing also needs to take into account compensatory mechanisms and other alterations that may not yet be characterized. Thus, testing for gene combinations may not paint a complete picture of DNA repair ability. Similarly, protein expression levels may not equate to DNA repair efficiency or deficiency. While it is important to identify the entire genome and epigenome of normal versus tumorigenic DNA repair, it may be even more important to understand the functional interactions of those pathways and their regulatory components. Functional repair assays, which measure how readily proteins can localize into foci, may provide a more accurate picture of repair activity, even without identifying every repair component [5].

\subsection{Identify and catalogue biomarkers for cancers}

This encompasses two realms: data and technologies. The right technologies must be used that can detect existing variants and find new combinations of variants. In addition, the results need to be quantified to assess whether the biomarkers have prognostic or predictive value. Testing, scoring, and cutoff points must be defined. 


\subsection{Correlate biomarker findings with DNA repair activities}

Our ability to correlate phenotypes to certain cancers has, to date, been limited by contradictory results from analyses of gene expression and polymorphisms, as well as studies that do not measure DNA repair capacity per se. Additional genetic and biochemical analyses are needed to determine the true clinical importance of individual genes, SNPs and certain groups of both [6].

\subsection{Make biomarker tests readily available}

The specialized equipment used in the research lab for such testing is impractical for clinical use. Accurate, specific, readily available tests need to be developed that can quickly identify defects in sensing or repairing DNA damage. Virtually every aspect of biomarker testingprotein extraction procedures, antibody selection, boosting detection limits, and moreneeds to be scrutinized in order to create and optimize clinically useful assays.

\subsection{Develop selective inhibitors against biomarkers}

There's no shortage of inhibitors in preclinical or clinical development, and the identification of more, new inhibitors is ongoing. However, a greater understanding of each DNA repair pathway is needed to determine which step and which protein will be most efficacious to inhibit.

\subsection{Perform patient testing for biomarkers as soon as cancer is identified}

Biomarker testing as a routine pretreatment step is predicated on the development of reliable, readily available testing methods as noted in bucket 5 . Such testing will help match patients with the treatments that will most likely yield the greatest benefit [7]. Pre-treatment biomarker testing also should also enable clinicians to start treatments sooner.

\subsection{Restructure clinical trials}

Clinical trials need to focus on the right treatment for each patient-not the average patient. Large, prospectively designed clinical trials that assess the impact and cost-effectiveness of pre-treatment genotyping approaches for targeted patient therapy [8] should yield better trial results overall, as well as results for individuals [9].

\subsection{Adjust anticancer regimens according to molecular profiles}

Adding molecular patient profiles to "traditional" demographics (age, gender, ethnicity, type and stage of cancer) as a basis for determining anticancer treatment regimens will increase the chances of treatment success. Such profiling could not only designate the right treatment, but also suggest new treatment regimens. Potential examples include using a DNA repair inhibitor (1) for premalignant lesions, (2) with other anticancer agents in intermittent dosing, (3) after ionizing radiation, and (4) coupled with another inhibitor at the end of a treatment regimen $[7,10-12]$.

\section{Concerns associated with DNA repair inhibitors}

Although the path of DNA damage response and repair inhibition holds great promise, it is also paved with concerns - the three main ones being (1) cytotoxicity, (2) secondary cancers, and (3) selecting for resistant cell populations.

Cytotoxicity, clinically manifested in rapidly proliferating normal tissues in the form of myelosuppression or gastrointestinal toxicity, almost always occurs when an inhibitor is given with a DNA-damaging drug. To maximize treatment benefit while minimizing collateral damage, numerous regimens are being tried, as already noted. 
A second concern is how to use DNA inhibitors without predisposing patients to secondary cancers. We already know that some BRCA-deficient cancers treated with a PARP inhibitor can develop additional mutations that restore the open reading frame and BRCA functionality $[4,10,12]$.

Third, targeting one gene may not only be toxic to healthy cells, but it also may "select out" resistant cell populations. As noted previously, it is likely that more than one gene is responsible for altered DNA repair abilities in many cancers, so finding reliable biomarkers and developing the right inhibitors are critical to successful DNA repair inhibition therapy.

\section{Meeting tomorrow's challenges}

Developing DNA repair inhibitors is challenging on many fronts. Characterizing DNA repair functions in cancerous lesions will drive the development and use of DNA-protein binding inhibitors as anticancer therapies and increase the chances of finding inhibitors that disrupt their targets in potent, specific, reproducible ways [7,11].

Researchers must prove that development of inhibitors is better stewardship of R\&D resources and more effective use of patients' and insurers' resources than non-specific treatments. A truly targeted treatment based on a patient's mutation profile (genome sequencing overlaid with other demographics), serves the patient better and makes R\&D efforts more fruitful. The inhibitors also must be safe as both single agents and adjunctive therapies - which necessitates concurrent single-agent and combination clinical trials [1]. This is not unique for DNA repair inhibitors.

\section{Summary}

We stand at an exciting inflection point regarding the use of disruptive approaches to DNA repair machinery in tumor cells. The challenge is to exploit them in a targeted manner that effectively augments our arsenal of weapons against cancer-particularly those cancers that represent treatment challenges and unmet needs, such as pancreatic and ovarian cancer, brain tumors, problematic pediatric cancers and others. This is especially critical for those that are orphan cancers not receiving significant efforts.

\section{Acknowledgments}

Financial support for this work was provided by the National Institutes of Health, National Cancer Institute CA121168, CA114571, and CA121168S1. Additional financial support was provided by the Riley Children's Foundation (Jeff Gordon Children's Foundation Laboratory) and the Betty and Earl Herr Chair in Pediatric Oncology Research. Also, thanks to Lana Christian of CreateWrite Inc., for her assistance and Julie Driscol for her administrative support and proofing.

\section{References}

1. Maxmen A. Beyond PARP inhibitors: agents in pipelines target DNA repair mechanisms. J. Natl. Cancer Inst. 2010; 102:1110-1111. [PubMed: 20668266]

2. Kelley, M. Future directions with DNA repair inhibitors: a roadmap for disruptive approaches to cancer therapy. In: Kelley, M., editor. DNA Repair in Cancer Therapy: Molecular Targets and Clinical Applications. Academic Press/Elsevier; 2012. p. 301-309.

3. Aziz K, Nowsheen S, Georgakilas AG. Nanotechnology in cancer therapy: targeting the inhibition of key DNA repair pathways. Curr. Mol. Med. 2010; 10:626-639. [PubMed: 20712589]

4. Rowe BP, Glazer PM. Emergence of rationally designed therapeutic strategies for breast cancer targeting DNA repair mechanisms. Breast Cancer Res. 2010; 12:203. [PubMed: 20459590] 
5. Willers, H.; Pfaffle, HN.; Zou, L. Targeting homologous recombination repair in cancer. In: Kelley, MR., editor. DNA Repair in Cancer Therapy: Molecular Targets and Clinical Applications. Academic Press/Elsevier; 2012. p. 119-151.

6. Shuck SC, Short EA, Turchi JJ. Eukaryotic nucleotide excision repair: from understanding mechanisms to influencing biology. Cell Res. 2008; 18:64-72. [PubMed: 18166981]

7. Helleday T, Petermann E, Lundin C, Hodgson B, Sharma RA. DNA repair pathways as targets for cancer therapy. Nat. Rev. 2008; 8:193-204.

8. Perry, C.; Sultana, R.; Madhusudan, S. Personalized cancer medicine: DNA repair alterations is a promising predictive marker in cancer. In: Kelley, MR., editor. DNA Repair in Cancer Therapy: Molecular Targets and Clinical Applications. Academic Press/Elsevier; 2012. p. 257-275.

9. de Bono JS, Ashworth A. Translating cancer research into targeted therapeutics. Nature. 2010; 467:543-549. [PubMed: 20882008]

10. Abbotts R, Madhusudan S. Human AP endonuclease 1 (APE1): from mechanistic insights to druggable target in cancer. Cancer Treat. Rev. 2010; 36:425-435. [PubMed: 20056333]

11. Gullotta F, De Marinis E, Ascenzi P, di Masi A. Targeting the DNA double strand breaks repair for cancer therapy. Curr. Med. Chem. 2010; 17:2017-2048. [PubMed: 20423312]

12. Plummer R. Perspective on the pipeline of drugs being developed with modulation of DNA damage as a target. Clin. Cancer Res. 2010; 16:4527-4531. [PubMed: 20823148] 